\title{
Loes Score
}

National Cancer Institute

\section{Source}

National Cancer Institute. Loes Score. NCI Thesaurus. Code C156449.

A rating of the severity of abnormalities in the brain found on MRI. It ranges from 0 to 34 , based on a point system derived from the location and extent of disease and the presence of atrophy in the brain, either localized to specific points or globally throughout the brain. 